\title{
Classification of the Flavobacterium-Cytophaga Complex on the Basis of Respiratory Quinones and Fumarate Respiration
}

\author{
EVELYN CALLIES AND W. MANNHEIM \\ Hygiene-Institut der Philipps-Universität, Marburg/Lahn, Federal Republic of Germany
}

\begin{abstract}
Respiratory quinones and the ability to use fumarate as a terminal electron acceptor in anaerobic respiration were investigated in 49 bacterial strains representing a variety of conventional Flavobacterium or Cytophaga species. The organisms examined were subdivided into two categories according to their quinones. (i) Ubiquinones are used by the neotype strain of Flavobacterium aquatile and by cultures representing $F$. acidificum, $F$. capsulatum, $F$. devorans, F. halmephilum, and some unnamed Flavobacterium species. (ii) Menaquinones are produced by both typical Cytophaga strains and many so-called Flavobacterium or "Flavobacterium/Cytophaga" cultures. Several members of category ii exhibited low to medium reduced nicotinamide adenine dinucleotide-fumarate reductase activities when grown in unaerated complex media supplemented with fumarate. In addition, with $F$. meningosepticum, "group IIb" organisms, and a strain of $F$. odoratum, the yields of oxygen-limited growth were markedly increased by fumarate, indicating an energetic use of fumarate respiration. On the basis of these findings, restriction of the genus Flavobacterium to "low-guanineplus-cytosine" organisms containing ubiquinones and resembling $F$. aquatile is proposed. The incorporation of some former "flavobacteria" into a natural group of organisms containing menaquinones and placement in the vicinity of the $C$. hutchinsonii guanine-plus-cytosine ratio are discussed.
\end{abstract}

The traditional genus Flavobacterium Bergey et al. consists of miscellaneous chemoorganotrophic bacteria that produce yellowish pigments. These organisms have proved to be highly heterogeneous regarding Gram reaction, type of motility, a variety of metabolic features, and, more recently, the base composition of their deoxyribonucleic acids (25). There appears to be general agreement that any attempt to reclassify these organisms would also have to consider the taxonomy of related groups, especially Cytophaga. This field has been termed the "Fla. vobacterium-Cytophaga complex" $(15,20)$.

The present study was initiated by the observation that Flavobacterium meningosepticum uses menaquinone in aerobic and anaerobic respiration, whereas $F$. aquatile contains ubiquinone as the sole respiratory quinone $(E$. Callies, unpublished data). Since, as a rule, quinqne patterns define well-established groups of chemoorganotrophic bacteria $(9,19)$, the taxonomic implications of this finding had to be considered. Therefore, the investigation was extended to a number of collection cultures representing several more-or-less recognized Flavobacterium or Cytophaga species. The data presented here show that quinones and quinone-mediated respiratory functions are very promising tools in defining natural groups of organisms contained in the Flavobacterium-Cytophaga complex.

\section{MATERIAIS AND METHODS}

Microorganisms. The bacterial strains investigated are listed in Table 1 . They were recovered from the lyophilic state by using appropriate media and conditions. Cloned subcultures were reidentified by a set of tests sufficient to rule out contamination and confusion, and propagated in mass cultures by using the media and growth conditions shown in Table 1.

Media. The following complex media were used and were supplemented with $50 \mathrm{mM}$ fumarate (disodium fumarate, sterilized by filtration; Fluka, Buchs, Switzerland) where indicated: (a) proteose peptone medium (26); (b) medium (a) supplemented with $0.25 \%$ (wt/vol) D-(t)-glucose (sterilized separately by heat in aqueous solution; E. Merck, Darmstadt, Germany); (c) medium (a) modified for the minerals sodium chloride $(0.8 \mathrm{M})$ and magnesium sulfate $(8 \mathrm{mM}$ ) (Merck chemicals); (d) medium (a) with $0.5 \mathrm{M}$ sodium chloride; (e) Trypticase soy broth (Baltimore Biological Laboratory, Cockeysville, Md.); (f) medium (e) with sodium chloride (final concentration, $2.65 \mathrm{M}$ ); (g) NCMB seawater medium (12); (h) tryptone-yeast extract-glucose medium according to McDermott et al. (14); (i) American Type Culture Collection medium 65; (k) medium (a) with sodium chloride (final concentration, $2 \mathrm{M}$ ) and supplemented with $0.2 \mathrm{M}$ potassium nitrate (25); (1) Dubos salts solution (12) supplemented with $5.0 \%$ (wt/vol) D-(+)-glucose. Although, before heat sterilization, some of the medium constituents contained varying amounts of lipoquinones, appropriate controls ensured that the results could not be affected by exogeneous quinones (16).

Cultivation. "Aerobic" cultures were grown in 
Fernbach flasks (no. 20511; Schott \& Gen., Mainz, Germany) containing $250 \mathrm{ml}$ of medium and aerated vigorously with a rotatory shaker (model G-25; New Brunswick Scientific Co., New Brunswick, N.J.). Oxygen-limited cultures were grown statically in Fernbach flasks filled up to the neck (ca. $2,000 \mathrm{ml}$ of medium). Late-exponential- or early-stationary-phase cells were harvested at $10,000 \times g, 0$ to $4^{\circ} \mathrm{C}$, and washed twice in $0.1 \mathrm{M}$ tris(hydroxymethyl)aminomethane-hydrochloride buffer, $\mathrm{pH}$ 7.2.

Disintegration of the cells, determination of protein, assay of NADH oxidase and of NADHfumarate reductase, and extraction and determination of quinones. Cells were disintegrated, protein was determined, reduced $\beta$-nicotinamide adenine dinucleotide (NADH) oxidase and NADH-fumarate reductase were assayed, and quinones were assayed and determined as described previously $(9,19)$.

Estimation of the effect of fumarate on growth densities in oxygen-limited cultures. Two 13-mmwide culture tubes containing $10 \mathrm{ml}$ of medium with or without added fumarate received small inocula from a preculture or suspension of the organism to be tested and were incubated statically at the individual growth temperature (see Table 1). Visual readings of the relative turbidities were done after 12,18 to 24 , and $48 \mathrm{~h}$, taking into consideration the possibility of a prolonged lag period with the cultures containing fumarate. For photometric recording, clear calibrated tubes were used, a tube that contained uninoculated medium serving as a blank.

\section{RESULTS}

Effect of fumarate on oxygen-limited growth. In unaerated tube cultures, most of the organisms studied grew poorly; among the organisms able to reach moderate growth densities in oxygen-limited cultures, Flavobacterium meningosepticum, the phenotypically related group IIb organisms, and $F$. odoratum (ATCC 4651) were unique in showing markedly increased growth responses after addition of fumarate to the complex medium. Essentially the same results were obtained with strictly anaerobic culture conditions. Therefore, quinone contents and electron transport activities of cells grown with oxygen or fumarate as an electron acceptor could be compared only in a minority of the strains investigated (see Table 1).

Quinones. The organisms representing Flavobacterium or Cytophaga species were found to contain either ubiquinones, between 0.3 and $2.2 \mu \mathrm{mol} / \mathrm{g}$ of cell protein (category i; Table 1A), or menaquinones, from 0.5 to about $5 \mu \mathrm{mol} / \mathrm{g}$ of cell protein (category ii; Table 1B), the amount of quinone produced by a given strain varying with culture conditions. Category i consists of several Flavobacterium species including the neotype strain of $F$. aquatile. Category ii contains the strains attributed to the genus Cytophaga, including the suggested working type of C. hutchinsonii, but also many so-called Flavo- bacterium cultures and, among them, those representing the $F$. meningosepticum group (i.e., serovars $A$ to $F$ and $E$. $O$. Kings group IIb).

Electron transport activities. NADH oxidase, at greatly varying activities, was present in all strains examined. Electron transport from NADH to fumarate, however, occurred only in some of the organisms containing menaquinones, namely, the $F$. meningosepticum group and the strains of $C$. hutchinsonii, $C$. johnsonae, $F$. odoratum, $F$. tirrenicum, and $F$. uliginosum examined. Thus, category ii is subdivided into two physiological groups capable, or not capable, of utilizing fumarate as a terminal acceptor in anaerobic electron transport.

\section{DISCUSSION}

If natural groups of chemoorganotrophic bacteria are characterized by their respiratory quinones, the data presented lead to the conclusion that the genus Flavobacterium should be restricted to organisms producing ubiquinones as the sole respiratory quinones and that the genus Cytophaga should comprise only organisms containing menaquinones. On the other hand, neither category i nor category ii is a homogeneous group of organisms, the base composition of their deoxyribonucleic acids ranging from below $30 \%$ to about $70 \%$ guanine plus cytosine $(G+C)$. It is likely that a homogeneous group can be detected among the nonfermentative, oxidasenegative, low- $\mathrm{G}+\mathrm{C}$ organisms containing ubiquinone and resembling $F$. aquatile (genus Flavobacterium sensu strictiori). Considering phenotypical characters and $\mathrm{G}+\mathrm{C}$ ratios, however, each of the other named species listed in Table $1 \mathrm{~A}$ appears to represent a particular taxonomic group distinct from Flavobacterium sensu strictiori. Category ii remains heterogeneous even after the gram-positive organisms ( $F$. arborescens, $F$. esteroaromaticum, $F$. flavescens, and $F$. suaveolens; Table 1B) are removed. Since a natural Cytophaga group (genus Cytophaga sensu strictiori) has to be restricted to a narrow range of $\mathrm{G}+\mathrm{C}$ ratios (4) situated in the vicinity of that of $C$. hutchinsonii, its relationship to the $F$. meningosepticum group (20) should be investigated, and the fate of both low-G+C and high$\mathrm{G}+\mathrm{C}$ strains outside this $\mathrm{G}+\mathrm{C}$ range (cf. Table 1B) remains open. Undoubtedly, the usefulness of the respiratory quinones as taxonomic criteria should be verified by investigating the deoxyribonucleic acid relatedness between selected members of the Flavobacterium-Cytophaga complex.

The data presented are in accordance with earlier reports on the quinones of Flavobacterium strain 0147 (7). The oxidase-positive $F$ lavobacterium strains of Denis et al. (6), contain- 
TABLE 1. Respiratory quinones, NADH oxidase, and NADH-fumarate reductase activities of some Flavobacterium and Cytophaga strains grown in complex media with oxygen or fumarate as the terminal electron acceptor (late-exponential-phase cells) ${ }^{a}$

\begin{tabular}{|c|c|c|c|c|c|c|c|}
\hline \multirow[t]{2}{*}{ Organism $^{b}$} & \multirow{2}{*}{$\begin{array}{c}\mathrm{G}+\mathrm{C} \text { content } \\
\text { of DNA } \\
\text { (mol\%) }\end{array}$} & \multirow{2}{*}{$\begin{array}{l}\text { Medium } \\
\text { and incu- } \\
\text { bation } \\
\text { temp }\left({ }^{\circ} \mathrm{C}\right) \\
\text { used for } \\
\text { mass cul- } \\
\text { tivation }\end{array}$} & \multirow{2}{*}{$\begin{array}{l}\text { Stimula- } \\
\text { tion of } \\
\text { oxygen- } \\
\text { limited } \\
\text { growth by } \\
\text { fumarate }\end{array}$} & \multirow{2}{*}{$\begin{array}{l}\text { Growth den- } \\
\text { sity }{ }^{d} \text { (g [dry } \\
\text { wt]/liter) }\end{array}$} & \multirow{2}{*}{$\begin{array}{l}\text { Quinones } \\
\text { (umol/g of } \\
\text { cell pro- } \\
\text { tein) }\end{array}$} & \multicolumn{2}{|c|}{$\begin{array}{l}\text { Electron transport } \\
\text { ( } \mu \text { mol of NADH oxi- } \\
\text { dized per g of protein } \\
\text { per min) to: }\end{array}$} \\
\hline & & & & & & Oxygen & Fumarate \\
\hline \multicolumn{8}{|l|}{$\begin{array}{l}\text { (A) Organisms containing ubi- } \\
\text { quinones }\end{array}$} \\
\hline $\begin{array}{l}\text { Flavobacterium aquatile, E. } \\
\text { W. Taylor F } 36 \text { (ATCC } \\
\text { 11947) }\end{array}$ & $32.0(25)^{e}$ & i, 25 & - & $\begin{array}{l}\text { (1) } 0.04 \\
(2,3) \text { NG }\end{array}$ & 0.35 & 9.4 & 0.0 \\
\hline $\begin{array}{l}\text { F. halmephilum, B. Elazari- } \\
\text { Volcani (ATCC 19717) }\end{array}$ & 49.7 (UD) & $\mathbf{k}, 30$ & - & $\begin{array}{l}\text { (1) } 0.70 \\
(2,3) \mathrm{NG}\end{array}$ & 1.56 & 9.4 & 0.0 \\
\hline $\begin{array}{c}\text { F. acidificum, E. A. Stein- } \\
\text { haus (ATCC 8366) }\end{array}$ & 52.7 (UD) & a, 30 & - & $\begin{array}{l}\text { (1) } 2.00 \\
\text { (2) } 0.03 \\
\text { (3) } 0.02\end{array}$ & $\begin{array}{l}0.57 \\
0.45 \\
0.35\end{array}$ & $\begin{array}{r}112.6 \\
64.6 \\
21.5\end{array}$ & $\begin{array}{l}0.0 \\
0.0 \\
0.0\end{array}$ \\
\hline $\begin{array}{l}\text { F. capsulatum, E. Leifson } 28 \\
\text { (ATCC 14666) }\end{array}$ & $63.0(20)$ & a, 30 & - & $\begin{array}{l}\text { (1) } 1.70 \\
(2,3) \text { NG }\end{array}$ & 1.20 & 4.4 & 0.0 \\
\hline$F \underset{\text { (ATCC 10829) }}{\text { devorans, N. Porges }}$ & $\begin{array}{l}69.0(25) \\
67.7 \text { (UD) }\end{array}$ & a, 30 & - & $\begin{array}{l}\text { (1) } 1.40 \\
(2,3) \mathrm{NG}\end{array}$ & 0.81 & 57.5 & 0.0 \\
\hline $\begin{array}{l}\text { Flavobacterium sp., L. S. } \\
\text { Suter K4 (ATCC 13945) }\end{array}$ & NT & a, 30 & - & $\begin{array}{l}\text { (1) } 2.65 \\
\text { (2) } 0.09 \\
\text { (3) } 0.09\end{array}$ & $\begin{array}{l}1.72 \\
2.15 \\
0.35\end{array}$ & $\begin{array}{r}50.9 \\
3.2 \\
4.2\end{array}$ & $\begin{array}{l}0.0 \\
0.0 \\
0.0\end{array}$ \\
\hline $\begin{array}{l}\text { Flavobacterium sp., Mar- } \\
\text { burg 19757/70 (HIM } 377 \text { - } \\
\text { 1) }\end{array}$ & NT & a, 30 & - & $\begin{array}{l}\text { (1) } 2.50 \\
(2,3) \mathrm{NG}\end{array}$ & 0.21 & 30.8 & 0.0 \\
\hline $\begin{array}{l}\text { Flavobacterium sp., Mar- } \\
\text { burg } 91100 / 74 \text { (HIM 484- } \\
\text { 7) }\end{array}$ & NT & a, 30 & - & $\begin{array}{l}\text { (1) } 0.76 \\
(2,3) \mathrm{NG}\end{array}$ & 0.57 & 4.1 & 0.0 \\
\hline $\begin{array}{l}\text { Flavobacterium sp., Mar- } \\
\text { burg } 91098 / 74 \text { (HIM } 484- \\
8 \text { ) }\end{array}$ & NT & a, 30 & - & $\begin{array}{l}\text { (1) } 1.00 \\
(2,3) \mathrm{NG}\end{array}$ & 0.59 & 30.0 & 0.0 \\
\hline $\begin{array}{l}\text { (B) Organisms containing } \\
\text { menaquinones }\end{array}$ & & & & & & & \\
\hline $\begin{array}{l}\text { F. breve, Ajinomoto Co., Inc. } \\
\text { S-15 (ATCC 14234) }\end{array}$ & $\begin{array}{l}26.0(25) \\
33.7(22)\end{array}$ & a, 30 & - & $\begin{array}{l}\text { (1) } 2.10 \\
(2,3) \mathrm{NG}\end{array}$ & 4.76 & 1.4 & 0.9 \\
\hline $\begin{array}{l}\text { F. uliginosum, C. E. ZoBell } \\
553 \text { (ATCC 14397) }\end{array}$ & $32.0(25)$ & $\mathrm{g}, 30$ & - & $\begin{array}{l}\text { (1) } 0.95 \\
(2,3) \mathrm{NG}\end{array}$ & 3.21 & 5.1 & 5.6 \\
\hline $\begin{array}{r}\text { pectinovorum, M. J. } \\
\text { Dorey, } 81 \text { (ATCC 19366) }\end{array}$ & $\begin{array}{l}32.9(20) \\
34.2(23) \\
34.9(22) \\
35.5 \text { (UD) }\end{array}$ & a, 30 & - & $\begin{array}{l}\text { (1) } 2.50 \\
(2,3) \mathrm{NG}\end{array}$ & 1.74 & 1.3 & 0.0 \\
\hline Cytophaga johnsonae, C. B. & $33-35(13)$ & a, 30 & - & (1) 2.70 & 1.82 & 16.5 & 0.0 \\
\hline $\begin{array}{l}\text { van Niel, MYX. 1.1.1. } \\
\text { (A'TCC 17061) }\end{array}$ & $33.0(14)$ & & & $\begin{array}{l}\text { (2) } 0.03 \\
\text { (3) } 0.02\end{array}$ & $\begin{array}{l}2.98 \\
0.56\end{array}$ & $\begin{array}{l}5.1 \\
2.6\end{array}$ & $\begin{array}{r}12.7 \\
0.0\end{array}$ \\
\hline $\begin{array}{l}\text { Flavobacterium sp., Hoff- } \\
\text { mann-La Roche \& Co. AG. } \\
\text { O147 (R 1519) }\end{array}$ & 33.9 (UD) & h, 20 & - & $\begin{array}{l}\text { (1) } 0.48 \\
\text { (2) } 0.01\end{array}$ & $\begin{array}{l}2.11 \\
2.00\end{array}$ & $\begin{array}{r}26.7 \\
2.7\end{array}$ & $\begin{array}{l}0.0 \\
0.0\end{array}$ \\
\hline$F . \quad$ tirrenicum, A. Grein & 34.4 (UD) & a, 30 & - & $\begin{array}{l}\text { (1) } 0.33 \\
\text { (2) } 0.01 \\
\text { (3) } 0.01\end{array}$ & $\begin{array}{l}0.90 \\
1.28 \\
1.09\end{array}$ & $\begin{array}{r}69.6 \\
351.1 \\
9.9\end{array}$ & $\begin{array}{r}17.8 \\
8.8 \\
6.7\end{array}$ \\
\hline $\begin{array}{l}\text { Flauobacterium/Cytophaga } \\
\text { sp., (5) (NCMB 251) }\end{array}$ & $35.6(5,18)$ & f, 24 & - & $\begin{array}{l}\text { (1) } 0.17 \\
(2,3) \mathrm{NG}\end{array}$ & 3.40 & 6.4 & 0.0 \\
\hline $\begin{array}{l}\text { Flavobacterium/Cytophaga } \\
\text { sp., (5) (NCMB 275) }\end{array}$ & $35.8(5,18)$ & a, 24 & - & $\begin{array}{l}\text { (1) } 0.17 \\
(2,3) \mathrm{NG}\end{array}$ & 1.04 & 9.2 & 0.7 \\
\hline $\begin{array}{l}\text { Flavobacterium/Cytophaga } \\
\text { sp., (5) (NCMB 289) }\end{array}$ & $\begin{array}{l}35.8(5) \\
35.5 \text { (UD) }\end{array}$ & c, 24 & - & $\begin{array}{l}\text { (1) } 0.95 \\
(2,3) \text { NG }\end{array}$ & 1.80 & 3.1 & 6.0 \\
\hline $\begin{array}{l}\text { Flavobacterium/Cytophaga } \\
\text { sp., (5) (NCMB 264) }\end{array}$ & $36.6(5,18)$ & c, 24 & - & $\begin{array}{l}\text { (1) } 1.40 \\
(2,3) \mathrm{NG}\end{array}$ & 2.49 & 15.5 & 0.0 \\
\hline
\end{tabular}


TABLE 1-Continued

\begin{tabular}{|c|c|c|c|c|c|c|c|}
\hline \multirow[t]{2}{*}{ Organism $^{b}$} & \multirow{2}{*}{$\begin{array}{l}\mathrm{G}+\mathrm{C} \text { content } \\
\text { of DNA } \\
\text { ( } \mathrm{mol} \%)\end{array}$} & \multirow{2}{*}{$\begin{array}{l}\text { Medium }^{c} \\
\text { and incu- } \\
\text { bation } \\
\text { temp }\left({ }^{\circ} \mathrm{C}\right) \\
\text { used for } \\
\text { mass cul- } \\
\text { tivation }\end{array}$} & \multirow{2}{*}{$\begin{array}{l}\text { Stimula- } \\
\text { tion of } \\
\text { oxygen- } \\
\text { limited } \\
\text { growth by } \\
\text { fumarate }\end{array}$} & \multirow{2}{*}{$\begin{array}{l}\text { Growth den- } \\
\text { sity }^{d}(\mathrm{~g}[\mathrm{dry} \\
\text { wt]/liter) }\end{array}$} & \multirow{2}{*}{$\begin{array}{l}\text { Quinones } \\
\text { ( } \mu \mathrm{mol} / \mathrm{g} \text { of } \\
\text { cell pro- } \\
\text { tein) }\end{array}$} & \multicolumn{2}{|c|}{$\begin{array}{l}\text { Electron transport } \\
\text { ( } \mu \text { mol of NADH oxi- } \\
\text { dized per g of protein } \\
\text { per min) to: }\end{array}$} \\
\hline & & & & & & Oxygen & Fumarate \\
\hline \multirow[t]{3}{*}{ F. odoratum, M. Stutzer } & $36.7(22)$ & a, 30 & ++ & (1) 1.20 & 1.83 & 31.2 & 22.0 \\
\hline & & & & (2) 0.42 & 2.01 & 78.8 & 95.5 \\
\hline & & & & (3) 0.16 & 1.22 & 29.6 & 15.1 \\
\hline \multirow{3}{*}{$\begin{array}{l}\text { F. meningosepticum, serovar } \\
\text { A, E. O. King (NCTC } \\
\text { 10016) }\end{array}$} & $35.5(24)$ & a, 30 & ++ & (1) 2.70 & 2.73 & 16.9 & 50.5 \\
\hline & $37.6(21)$ & & & (2) 0.57 & 4.50 & 22.6 & 279.4 \\
\hline & $\begin{array}{l}38.3(18,20) \\
38.4 \text { (UD) }\end{array}$ & & & (3) 0.01 & 1.58 & 6.4 & 12.8 \\
\hline \multirow{2}{*}{$\begin{array}{l}\text { F. meningosepticum, serovar } \\
\text { B, R. E. Weaver (NCTC } \\
\text { 10585) }\end{array}$} & $36.4(20)$ & a, 30 & ++ & (1) 1.10 & 2.80 & 2.8 & 4.0 \\
\hline & $\begin{array}{l}36.8(21) \\
50.0(24)\end{array}$ & & & (2) 0.06 & 2.44 & 8.9 & 14.0 \\
\hline \multirow{2}{*}{$\begin{array}{l}\text { F. meningosepticum, serovar } \\
\text { C, R. E. Weaver (NCTC } \\
\text { 10586) }\end{array}$} & $36.4(20)$ & a, 30 & ++ & (1) 1.11 & 3.37 & 3.4 & 10.4 \\
\hline & $\begin{array}{l}37.2(21) \\
37.5(24)\end{array}$ & & & (2) 0.04 & 2.23 & 13.2 & 42.8 \\
\hline \multirow{2}{*}{$\begin{array}{l}\text { F. meningosepticum, serovar } \\
\text { D, S. N. Arseculeratne } \\
\text { (NCTC 10587) }\end{array}$} & $36.8(21)$ & a, 30 & ++ & (1) 0.92 & 2.11 & 1.3 & 3.5 \\
\hline & $38.0(24)$ & & & (2) 0.03 & 2.06 & 3.9 & 11.8 \\
\hline \multirow{2}{*}{$\begin{array}{l}\text { F. meningosepticum, serovar } \\
\text { E, L. Allen (NCTC 10588) }\end{array}$} & $36.1(21)$ & a, 30 & ++ & (1) 1.10 & 1.80 & 1.7 & 2.1 \\
\hline & $39.0(24)$ & & & (2) 0.05 & 1.62 & 10.5 & 0.0 \\
\hline \multirow{2}{*}{$\begin{array}{l}\text { F. meningosepticum, serovar } \\
\text { F, A. Blevins (NCTC } \\
\text { 10589) }\end{array}$} & $31.6(24)$ & a, 30 & ++ & (1) 1.00 & 1.97 & 17.0 & 5.0 \\
\hline & $37.0(21)$ & & & (2) 0.05 & 2.45 & 13.8 & 14.4 \\
\hline \multirow{2}{*}{$\begin{array}{l}\text { Flavobacterium sp., group } \\
\text { IIb (21) (NCTC 10795) }\end{array}$} & $38.5(21)$ & a, 30 & ++ & (1) 2.50 & 1.20 & 13.0 & 18.7 \\
\hline & & & & (2) 0.11 & 2.43 & 13.6 & 8.4 \\
\hline \multirow{2}{*}{$\begin{array}{l}\text { Flavobacterium sp., group } \\
\text { IIb (21) (NCTC 10796) }\end{array}$} & $39.4(22)$ & a, 30 & ++ & (1) 2.10 & 1.45 & 19.1 & 46.2 \\
\hline & & & & (2) 0.19 & 1.35 & 20.7 & 42.5 \\
\hline \multirow{2}{*}{$\begin{array}{l}\text { Flavobacterium sp., group } \\
\text { IIb (21) (NCTC 10797) }\end{array}$} & $36.1(22)$ & a, 30 & + & (1) 1.74 & 0.77 & 13.1 & 14.6 \\
\hline & & & & (2) 0.01 & 0.88 & 13.6 & 6.9 \\
\hline \multirow{2}{*}{$\begin{array}{l}\text { Flavobacterium sp., group } \\
\text { IIb, Marburg } 5388 / 69 \\
\text { (HIM 326-8) }\end{array}$} & NT & a, 30 & + & (1) 1.70 & 1.40 & 2.0 & 0.4 \\
\hline & & & & (2) 0.23 & NT & 7.0 & 5.6 \\
\hline \multirow{2}{*}{$\begin{array}{l}\text { Flavobacterium sp., group } \\
\text { IIb, Marburg } 153421 / 71 \\
\text { (HIM 418-5) }\end{array}$} & NT & a, 30 & ++ & (1) 1.41 & 2.18 & 15.2 & 21.8 \\
\hline & & & & (2) 0.19 & 3.03 & 3.1 & 7.1 \\
\hline \multirow{2}{*}{$\begin{array}{l}\text { Flavobacterium sp., group } \\
\text { IIb, Marburg } 141230 / 73 \\
\text { (HIM 496-4) }\end{array}$} & NT & a, 30 & + & (1) 2.53 & 2.12 & 9.3 & 2.3 \\
\hline & & & & (2) 0.18 & NT & 5.6 & 4.9 \\
\hline \multirow{2}{*}{$\begin{array}{l}\text { Flavobacterium sp., group } \\
\text { IIb, Marburg 190568/73 } \\
\text { (HIM 464-2) }\end{array}$} & NT & a, 30 & + & (1) 2.30 & 4.11 & 8.3 & 4.4 \\
\hline & & & & (2) 0.23 & 1.40 & 8.2 & 3.3 \\
\hline \multirow{2}{*}{$\begin{array}{l}\text { Flavobacterium sp., group } \\
\text { IIb, Marburg } 14135 / 74 \\
\text { (HIM 494-3) }\end{array}$} & NT & a, 30 & ++ & (1) 2.65 & 4.76 & 15.3 & 14.3 \\
\hline & & & & (2) 0.16 & 7.51 & 15.0 & 5.5 \\
\hline \multirow{2}{*}{$\begin{array}{l}\text { Flavobacterium sp., group } \\
\text { IIb, Marburg } 54552 / 74 \\
\text { (HIM 482-9) }\end{array}$} & NT & a, 30 & + & (1) 2.13 & 3.05 & 5.2 & 1.3 \\
\hline & & & & (2) 0.15 & NT & 2.7 & 1.4 \\
\hline Flavobacterium sp., group & NT & a, 30 & (Floccu- & (1) 1.70 & 2.47 & 5.3 & 4.6 \\
\hline $\begin{array}{l}\text { IIb, Marburg 123496/74 } \\
\text { (HIM 488-5) }\end{array}$ & & & lent) & (2) 0.02 & 2.74 & 7.9 & 5.6 \\
\hline $\begin{array}{l}\text { C. marinoflava, R. Spencer } \\
\text { (ATCC 19326) }\end{array}$ & $37.0(3)$ & $c, 30$ & - & $\begin{array}{l}\text { (1) } 0.93 \\
(2,3) \mathrm{NG}\end{array}$ & 1.61 & 2.8 & 1.6 \\
\hline $\begin{array}{l}\text { C. hutchinsonii, R. Y. Stan- } \\
\text { ier, 9-37 (NCIB 9469) }\end{array}$ & $39.0(14)$ & 1,25 & - & $\begin{array}{l}\text { (1) } 0.42 \\
(2,3) \text { NG }\end{array}$ & 0.81 & 8.5 & 15.0 \\
\hline C. fermentans, H. Veldkamp & $41.2(14)$ & a, 30 & - & (1) 0.29 & 0.66 & 7.1 & 0.0 \\
\hline (ATCC 19072) & & & & (2) 0.02 & 0.91 & 4.1 & 0.0 \\
\hline & & & & (3) 0.02 & 0.89 & 8.8 & 0.0 \\
\hline $\begin{array}{l}\text { Flavobacterium/Cytophaga } \\
\text { sp., (5) (NCMB 249) }\end{array}$ & $40.6(5)$ & a, 24 & - & $\begin{array}{l}\text { (1) } 0.05 \\
(2,3) \text { NG }\end{array}$ & 0.79 & 5.1 & 0.9 \\
\hline
\end{tabular}




\begin{tabular}{|c|c|c|c|c|c|c|c|}
\hline \multirow[t]{2}{*}{ Organism $^{b}$} & \multirow{2}{*}{$\begin{array}{c}\mathrm{G}+\mathrm{C} \text { content } \\
\text { of DNA } \\
(\mathrm{mol} \%)\end{array}$} & \multirow{2}{*}{$\begin{array}{l}\text { Medium } \\
\text { and incu- } \\
\text { bation } \\
\text { temp }\left({ }^{\circ} \mathrm{C}\right) \\
\text { used for } \\
\text { mass cul- } \\
\text { tivation }\end{array}$} & \multirow{2}{*}{$\begin{array}{l}\text { Stimula- } \\
\text { tion of } \\
\text { oxygen- } \\
\text { limited } \\
\text { growth by } \\
\text { fumarate }\end{array}$} & \multirow{2}{*}{$\begin{array}{l}\text { Growth den- } \\
\text { sity }^{d} \text { (g [dry } \\
\text { wt]/liter) }\end{array}$} & \multirow{2}{*}{$\begin{array}{l}\text { Quinones } \\
\text { (umol/g of } \\
\text { cell pro- } \\
\text { tein) }\end{array}$} & \multicolumn{2}{|c|}{$\begin{array}{l}\text { Electron transport } \\
\text { ( } \mu \text { mol of NADH oxi- } \\
\text { dized per g of protein } \\
\text { per min) to: }\end{array}$} \\
\hline & & & & & & Oxygen & Fumarate \\
\hline $\begin{array}{l}\text { C. aurantiaca, H. Bortels } \\
\text { (ATCC 12208) }\end{array}$ & $42.0(14)$ & a, 30 & - & $\begin{array}{l}\text { (1) } 2.53 \\
(2,3) \text { NG }\end{array}$ & 1.00 & 16.5 & 0.0 \\
\hline $\begin{array}{l}F . \text { heparinum, E. K. Korn } \\
\text { (NIH) (ATCC 13125) }\end{array}$ & $\begin{array}{l}42.2(20) \\
44.7(22) \\
45.0(\mathrm{UD}) \\
45.6(23)\end{array}$ & a, 30 & - & $\begin{array}{l}\text { (1) } 0.76 \\
(2,3) \mathrm{NG}\end{array}$ & 1.15 & NT & NT \\
\hline $\begin{array}{l}F . \text { ferrugineum, Julia M. } \\
\text { Coffey } 3576 \text { (ATCC 13524) }\end{array}$ & $42.6(25)$ & a, 30 & - & $\begin{array}{l}\text { (1) } 0.27 \\
(2,3) \text { NG }\end{array}$ & 0.65 & 1.4 & 1.0 \\
\hline $\begin{array}{l}\text { Flavobacterium/Cytophaga } \\
\text { sp., (5) (NCMB 244) }\end{array}$ & $62.9(5,18)$ & b, 24 & - & $\begin{array}{l}\text { (1) } 1.55 \\
(2,3) \mathrm{NG}\end{array}$ & 1.33 & 1.6 & 0.0 \\
\hline $\begin{array}{c}\text { Flavobacterium/Cytophaga } \\
\text { sp., (5) (NCMB 259) }\end{array}$ & $63.7(5,18)$ & b, 24 & - & $\begin{array}{l}\text { (1) } 2.30 \\
(2,3) \mathrm{NG}\end{array}$ & 0.91 & 45.6 & 0.0 \\
\hline $\begin{array}{c}F \text { esteroaromaticum, C. P. } \\
\text { Hegarty (ATCC 8091) }\end{array}$ & $69.0(25)$ & a, 30 & - & $\begin{array}{l}\text { (1) } 2.54 \\
(2,3) \mathrm{NG}\end{array}$ & 0.93 & 8.5 & 0.0 \\
\hline $\begin{array}{l}\text { F. arborescens, A. Stewart } \\
\text { (ATCC 4358) }\end{array}$ & $71.0(25)$ & a, 30 & - & $\begin{array}{l}\text { (1) } 0.24 \\
(2,3) \mathrm{NG}\end{array}$ & 0.50 & 2.4 & 0.0 \\
\hline $\begin{array}{l}F \text {. flavescens, C. Darling } 72 \\
\text { (ATCC 8315) }\end{array}$ & $71.0(25)$ & a, 30 & - & $\begin{array}{l}\text { (1) } 1.41 \\
(2,3) \mathrm{NG}\end{array}$ & 0.94 & 4.0 & 0.0 \\
\hline $\begin{array}{c}F . \text { suaveolens, M. Levine } \\
1045 \text { (1) (ATCC 958) }\end{array}$ & $70.0(25)$ & a, 30 & - & (1) 2.50 & 1.23 & 10.1 & 0.0 \\
\hline
\end{tabular}

a Abbreviations: ATCC, American Type Culture Collection, Rockville, Md.; HIM, culture collection of the Hygiene-Institut der Philipps-Universität Marburg, Germany; NCIB, National Collection of Industrial Bacteria, Torry Research Station, Aberdeen, Scotland; NCMB, National Collection of Marine Bacteria, Torry Research Station, Aberdeen, Scotland; NCTC, National Collection of Type Cultures, Public Health Laboratory, London, England; R, Culture Collection of the Hoffmann-La Roche \& Co. AG, Basel, Switzerland. DNA, deoxyribonucleic acid; NG, no growth; NT, not tested; UD, unpublished data of E. Callies.

${ }^{b}$ Strain designations, according to the culture collection catalogues, most recent editions, or personal communications (HIM, R, respectively).

' For compositions of media, see Materials and Methods.

d 1 , Oxygen; 2 , fumarate; 3 , no added acceptor.

Reference.

ing $Q-8, Q-9$, or $Q$-indeterminate ( $Q$ represents ubiquinone), are probably different from $F$. aquatile, which is oxidase negative, the length of the isoprenoid side chains of the quinones not being considered in the present communication. Some organisms considered to be related to Cytophaga, like the flexibacters and myxobacters, contain menaquinones (10; E. Callies, unpublished data). However, other organisms thought to be more or less closely related to Cytophaga, such as Beggiatoa and Vitreoscilla, contain only ubiquinones when cultivated under conditions similar to those used in the present study (Callies, unpublished data); the Beggiatoa culture investigated by Carr and co-workers (2) produced naphthoquinones in addition to ubiquinone.

The growth stimulation by fumarate in unaerated proteose peptone cultures observed with organisms of the $F$. meningosepticum group deserves attention in diagnostic bacteriology, since this phenomenon indicates the presence of naphthoquinone-mediated anaerobic electron transport. Anaerobic fumarate respiration is also characteristic of the Enterobacteriaceae, the Actinobacillus, Haemophilus, and Pasteurella groups, and some Bacteroidaceae (9; unpublished data). The NADH-fumarate reductase system of $F$. meningosepticum involves $b$-type cytochromes and menaquinone and is membrane bound (E. Callies, manuscript in preparation), thus resembling that of Proteus rettgeri (11). The NADH-fumarate reductase activities reported do not necessarily account for the extent of anaerobic electron transport, since other donors that may be used, such as formate or hydrogen, were not taken into consideration. The presence of presumably membrane-bound NADH-fumarate reductases in some Flavobacterium and Cytophaga strains lacking the growth response to fumarate in anaerobic complex media cannot be discussed here in detail. Regarding the similarity of $F$. acidificum and Erwinia herbicola (20), the absence of NADH- 
fumarate reductase in the former (ATCC 8366; Table 1A) should be noted, since $E$. herbicola is able to produce a highly active, quinone-free, soluble NADH-fumarate reductase system (W. Mannheim and co-workers, unpublished data).

\section{REPRINT REQUESTS}

Address reprint requests to: Dr. W. Mannheim, HygieneInstitut der Philipps-Universität, D-3550 Marburg/Lahn, Pilgrimstein 2, Federal Republic of Germany.

\section{LTERATURE CITED}

1. American Type Culture Collection. 1974. Catalogue of strains, 11th ed. American Type Culture Collection, Rockville, Md.

2. Carr, N. G., G. Brell, V. Flynn, M. Hallaway, and S. Talukdar. 1967. Minor quinones of some myxophyceae. Arch. Biochem. Biophys. 120:503-507.

3. Colwell, R. R., R. V. Citarella, and P. K. Chen. 1966. DNA base composition of Cytophaga marinoflava $n$. sp. determined by buoyant density measurements in cesium chloride. Can. J. Microbiol. 12:1099-1103.

4. DeLey, J. 1989. Compositional nucleotide distribution and the theoretical prediction of homology in bacterial DNA. J. Theor. Biol. 22:89-116.

5. DeLey, J., and J. van Muylem. 1963. Some applications of deoryribonucleic acid base composition in bacterial taxonomy. Antonie van Leeuwenhoek J. Microbiol. Serol. 28:344-358.

6. Denis, F. A., P. A. D'Qultremont, J. J. Debagq, J. M. Cherel, and J. Brisou. 1975. Distribution des ubiquinones (coenzyme Q) chez les bacilles à Gram négatif. C. R. Soc. Biol. 2:380-382.

7. Dunphy, P. J., P. G. Phillips, and A. F. Brodie. 1971. Separation and identification of menaquinones from microorganisms. J. Lipid Res. 12:442-449.

8. Edelman, M., D. Swinton, J. A. Schiff, H. T. Epstein, and B. Zeldin. 1967. Deoxyribonucleic acid base of the blue-green algae (Cyanophyta). Bacteriol. Rev. $31: 315-331$.

9. Holländer, R., and W. Mannheim. 1975. Characterization of hemophilic and related bacteria by thein respiratory quinones and cytochromes. Int. J. Syst. Bacteriol. 25:102-107.

10. Kleinig, H., H. Reichenbach, N. Theobald, and $H$. Achenbach. 1974. Flexibacter elegans and Myxococcus fulvus: aerobic gram-negative bacteria containing menaquinones as the only isoprenoid quinones. Arch. Microbiol. 101:91-93.

11. Kröger, A., V. Dadák, M. Klingenberg, and F. Diemer. 1971. On the role of quinones in bacterial electron transport. Eur. J. Biochem. 21:322-333.
12. Lapage, S. P., J. E. Shelton, and T. G. Mitchell. 1970 Media for the maintenance and preservation of bacteria, p. 1-133. In J. R. Norris and D. W. Ribbons (ed.), Methods in microbiology, vol. 3A. Academic Press Inc., London

13. Leadbetter, E. R. 1974. Genus I. Cytophaga Winogradsky 1929, p. 101-105. In R. E. Buchanan and N. E Gibbons (ed.), Bergey's manual of determinative bacteriology, 8th ed. The Williams \& Wilkins Co., Baltimore.

14. McDermott, J. C. B., G. Britton, and T. W. Godwin. 1973. Effect of inhibitors on zeaxanthin synthesis in a Flavobacterium. J. Gen. Microbiol. 77:161-171.

15. McMeekin, T. A., J. T. Patterson, D. B. Stewart, and J. G. Murray. 1973. The metabolism of amino compounds by some gram negative yellow pigmented rods. J. Appl. Bacteriol. 36:101-108.

16. McMeekin, T. A., D. B. Stewart, and J. G. Murray. 1972. The adansonian taxonomy and the deoxyribonucleic acid base composition of some gram-negative, yellow pigmented rods. J. Appl. Bacteriol. 35:129-137.

17. Mandel, M., and E. R. Leadbetter. 1965. Deoxyribonucleic acid base composition of myxobacteria. J. Bacteriol. 90:1795-1796.

18. Mandel, M., and R. A. Lewin. 1969. Deoxyribonucleic acid base composition of flexibacteria. J. Gen. Microbiol. 58:171-178.

19. Mannheim, W., W. Stieler, G. Wolf, and R. Zabel. 1978. Taxonomic significance of respiratory quinones and fumarate respiration in Actinobacillus and Pas. teurella. Int. J. Syst. Bacteriol. 28:7-13.

20. Mitchell, T. G., M. S. Hendrie, and J. M. Shewan. 1969. The taxonomy, differentiation and identification of Cytophaga species. J. Appl. Bacteriol. 32:40-50.

21. Owen, R. J., and S. P. Lapage. 1974. A comparison of strains of King's group IIb of Flavobacterium with Flavobacterium meningosepticum. Antonie van Leeuwenhoek J. Microbiol. Serol. 40:255-264.

22. Owen, R. J., and J. J. S. Snell. 1976. Deoxyribonucleic acid reassociation in the classification of flavobacteria. J. Gen. Microbiol. 93:89-102.

23. Perry, L. B. 1973. Gliding motility in some non-spreading flexibacteria. J. Appl. Bacteriol. 36:227-232.

24. Sottile, M. J., J. N. Baldwin, and R. E. Weaver. 1973. Deoxyribonucleic acid hybridization studies on Flavobacterium meningosepticum. Appl. Microbiol. 26: 535-539.

25. Weeks, O. B. 1974. Genus Flavobacterium Bergey et al. 1923, p. 357-364. In R. E. Buchanan and N. E. Gibbons (ed.), Bergey's manual of determinative bacteriology, 8th ed. The Williams \& Wilkins Co., Baltimore.

26. White, D. C., and L. Smith. 1962. Hematin enzymes of Haemophilus parainfluenzae. J. Biol. Chem. 237:1332-1336. 